\title{
RADIATION OF ELECTRON IN THE FIELD OF PLANE LIGHT WAVE*
}

\author{
I. Drebot", Yu. Grigor'ev, A. Zelinsky, O. Zvonar'ova, National Science Center "Kharkov Institute \\ of Physics and Technology", Kharkov, Ukraine \\ R. Tatchyn, SSRL, SLAC, Stanford, U.S.A.
}

\begin{abstract}
Results of integration of a Lorentz equation for a relativistic electron moving in the field of running, plane, linear polarized electromagnetic wave are presented in the paper. It is shown that electron velocities in the field of the wave are almost periodic functions of time.

For calculations of angular spectrum of electron radiation intensity expansion of the electromagnetic field in a wave zone into generalized Fourier series was used. Expressions for the radiation intensity spectrum are presented in the paper. Derived results are illustrated for electron and laser beam parameters of NSC KIPT X-ray generator NESTOR. It is shown that for low intensity of the interacting electromagnetic wave the results of energy and angular spectrum calculations in the frame of classical electrodynamics completely coincide with calculation results produced using quantum electrodynamics. Simultaneously, derived expressions give possibilities to investigate dependence of energy and angular Compton radiation spectrum on phase of interaction and the interacting wave intensity.
\end{abstract}

\section{INTRODUCTION}

Today a method of generation of intense, short-wave radiation under interaction of an intense electromagnetic wave with relativistic electrons moving toward the wave front (Compton backscattering) is widely discussed [1,2]. Analytical investigations of the process can be carried out both as well with quantum electrodynamics as with classical electrodynamics approaches. According to the generally accepted quantum electrodynamics approach interaction of an electron with an electromagnetic wave is described by KleinNishina formula which is applicable only for an interacting wave with low intensity [3]. In other words, the model is adequate when one electron interacts simultaneously only with one photon. If external field is more intensive (the number of photons in an oscillation mode $N_{p h}$ are $\gg 1$ ) the further derivation has to be produced. At the limit (at $N_{p h} \rightarrow \infty$ ), according to Bohr correspondence principle, scattering of an electromagnetic wave on an electron has to be described as well with quantum as with classical electrodynamics. However, it is worth to note, that development of theory of interaction of an intense electromagnetic wave with an electron is very time-consuming and complicated way [see for example 4]. At the same time, as it seems to us, using of the classical electrodynamics approach allows to consider the task with simpler methods and to investigate as well an electron dynamics in the field of external electromagnetic field as effect of the external field intensity on spectrum and intensity of the generated radiation.

\footnotetext{
*Work supported by NATO SfP \#977982 grand.

\#drebot@kipt.kharkov.ua
}

\section{ELECTRON TRAJECTORIES IN THE FIELD OF ELECTROMAGNETIC WAVE}

Let us consider a relativistic electron with energy $E_{0}$, moving in laboratory coordinate frame under angles $\alpha_{1}, \alpha_{2}, \alpha_{3}$ toward plane, linear polarized, electromagnetic wave with field intensity $E_{\lambda}$. The wave is running along $x$ axis (Fig. 1). $\varphi$ and $\theta$ are polar angles to an observer.

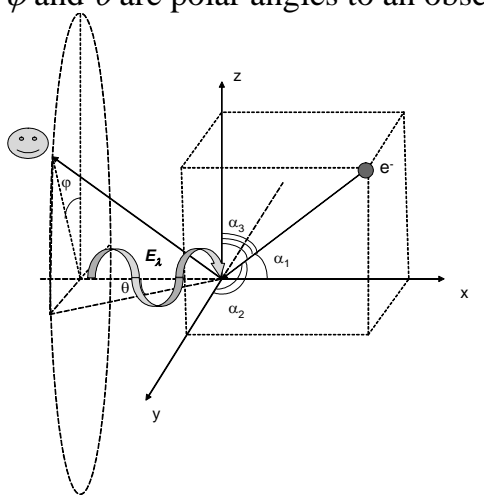

Figure 1: The scheme of Compton scattering.

A Lorentz equation for the electron can be written in the following form:

$$
\frac{d}{d t} m \vec{v}=c \vec{E}+\frac{e}{c}[\vec{v} \vec{H}]
$$

where $m=m_{0} /\left(1-\beta^{2}\right)^{1 / 2}, \beta=v / c, m_{0}$ is the rest mass of the electron, $c$ is velocity of light, $e$ is electron charge, $\vec{v}$ is an electron velocity vector, $t$ is time, $\vec{E}, \vec{H}$ are vectors of electric and magnetic field.

Electrical component of the field can be written as:

$$
E_{z}=E \cos \left[2 \pi v\left(t-\frac{x}{c}\right)+\delta\right],
$$

where $v$ is the field frequency, $\delta$ is an initial value of the wave phase relative to the interacting electron, $E_{z}$ is projection of electrical components of the field onto axis $z$. Other projections are $E_{x} \equiv 0, E \equiv 0$. Let normal to the front of the running wave $\vec{n}$ will have direction which coincides with $x$ axis.

Vector of magnetic field is:

$$
\vec{H}=[\vec{n} \vec{E}],
$$

where $\vec{n}=\vec{i}, \vec{i}$ is unit vector of $x$ axis.

After projecting (1) onto coordinate frame axes each out of three projections can be integrated at least one time and as a result we obtain:

$\beta_{x t}=\frac{F^{2}+1-B^{2}}{F^{2}+1+B^{2}}, \quad \beta_{z t}=\frac{2 B F}{F^{2}+1+B^{2}}, \beta_{y t}=\frac{2 B}{F^{2}+1+B^{2}}(4-6)$ 
where $F=P \sin \left[2 \pi v\left(t-\frac{x}{c}\right)+\delta\right]+N$,

$P=\frac{(e E / 2 \pi v)}{\left(m_{0}{ }^{2} c^{2}+a^{2}\right)^{1 / 2}}, \quad a=\frac{m_{0} c \beta_{y t}(0)}{\left(1-\beta^{2}(0)\right)}$,

$B=\frac{1-\beta_{x t}(0)}{\left(1-\beta_{x t}^{2}(0)-\beta_{z t}^{2}(0)\right)^{1 / 2}}=$ Const,

$N=\frac{m_{0} c \beta_{z t}(0)}{\sqrt{1-\beta^{2}(0) \sqrt{\left(m_{0} c\right)^{2}+a^{2}}}}-P \sin \left[2 \pi v s_{0}+\delta\right], s_{0}=t_{0}-\frac{x(0)}{c}$.

In integration the following formula was used:

$$
\frac{d \varepsilon}{d t}=e \frac{d z}{d t} E \cos \left[2 \pi v\left(t-\frac{x}{c}\right)+\delta\right] .
$$

For (1) integration we introduce new variable:

$$
g=2 \pi v\left(t-\frac{x}{c}\right)+\delta .
$$

Introducing notations:

$\beta_{x}(g)=\frac{1}{c} \frac{d x}{d g}, \beta_{z}(g)=\frac{1}{c} \frac{d z}{d g}, \beta_{y}(g)=\frac{1}{c} \frac{d y}{d g}$.

And substituting (9) into (4-6), we obtain:

$\beta_{x}(g)=\frac{1}{2 B^{2}(2 \pi v)}\left(1+F(g)^{2}-B^{2}\right), \beta_{z}(g)=\frac{1}{2 \pi v B} F(g)$,

$\beta_{y}(g)=\frac{1}{2 \pi v B} \frac{a}{\sqrt{\left(m_{0} c\right)^{2}+a^{2}}}$.

Integrating, we obtain the following:

$\frac{1}{c} x(g)=\frac{1}{4 \pi v B^{2}}\left(1-B^{2}+\frac{1}{2} P^{2}+N^{2}\right) g$

$-\frac{1}{4 \pi v B^{2}}\left(2 P N-\frac{1}{2} P^{2} \sin [g] \cos [g]\right)$

$\frac{1}{c} z(g)=\frac{1}{2 \pi v B} N g-\frac{1}{2 \pi \nu B} P \cos [g]$,

$\frac{1}{c} y(g)=\frac{a}{2 \pi v B \sqrt{m_{0}^{2} c^{2}+a^{2}}}$,

It is seen from expressions (10) that components of electron velocity are rational functions of function $F$, which satisfies a wave equation. Expressions (11-13) completely describe an electron motion in the field of electromagnetic wave. As one can see electron trajectories are not just "SIN" or "COS" like trajectories as it was supposed for example in [5], but have a strong dependence on intensity of an interacting wave, phase and angles of interaction.

\section{PARAMETERS OF GENERATED RADIATION}

It is known that functions satisfying a wave equation are almost periodical functions of $t$ in Hilbert space. So, we can make obvious supposition that rational functions of almost periodical function are almost periodical functions of time too. And, as it is known from Fourier series theory, almost periodical functions can be expanded in generalized Fourier series.

Thus, magnetic component of the field radiated by the electron on far distance (distance is longer then radiation wavelength) can be expressed in the following form [6]:

$\vec{H}=\sum_{f} H_{\omega f} e^{-i \omega_{f} t}$,

where $\vec{H}_{\omega f}=\frac{2 e i \omega_{f} e^{i k R_{0}}}{c^{2} R_{0}} \lim _{T \rightarrow \infty} \frac{1}{T} \int_{0}^{T} e^{i \omega_{f}\left(t-\frac{\vec{n} \vec{r}_{0}}{c}\right)}[\vec{n} d \vec{r}(t)]$,

$R_{0}$ is distance from point of origin to point of observation, $\vec{n}$ is unit vector in the same direction, $\omega_{f}$ is frequency of the radiation harmonic, $\vec{k}=\left(\omega_{f} / c\right) \vec{n}$ is the wave vector.

For $\vec{H}_{\omega f}$ calculating it is needed to change over from time $t$ in expression (14) to parameter $g$ (8). After change of variables we obtain:

$\vec{H}_{\omega f}=A \frac{e i \omega_{f} e^{i k R_{0}}}{c^{2} R_{0}} \lim _{g \rightarrow \infty} \frac{1}{g} \int_{0}^{g} e^{i \omega_{f}(\Lambda g+(\Psi-\aleph \operatorname{Sin}[g]) \operatorname{Cos}[g])}[\vec{n} \vec{\beta}(g)] d g$,

where $\Lambda=\frac{1}{4 \pi \nu}+\frac{1}{4 B^{2} \pi \nu}+\frac{P^{2}}{8 B^{2} \pi \nu}+\frac{\tilde{N}^{2}}{4 B^{2} \pi \nu}+$

$\left(\frac{1}{4 \pi v}-\frac{1}{4 B^{2} \pi v}-\frac{P^{2}}{8 B^{2} \pi v}-\frac{\tilde{N}^{2}}{4 B^{2} \pi v}\right) \cos \left(\gamma_{1}\right)-$

$\frac{\tilde{N}}{2 B \pi \nu} \cos \left(\gamma_{2}\right)-\frac{a \cos \left(\gamma_{3}\right)}{2 B \pi v \sqrt{a^{2}+m^{2} c^{2}}}$,

$\Psi=\left(\frac{\cos \left(\gamma_{2}\right)}{2 B \pi \nu}-\frac{\tilde{N}}{2 B^{2} \pi \nu}+\frac{\cos \left(\gamma_{1}\right) \tilde{N}}{2 B^{2} \pi \nu}\right) P$,

$\chi=\frac{P^{2}}{8 B^{2} \pi v}\left(1-\cos \left(\gamma_{1}\right)\right)$.

Expanding (15) one can find harmonic frequencies $\omega_{f}=f / \Lambda$, where $f$ is integer.

The harmonic radiation energy one can find from the following expression:

$$
\varepsilon_{p h}=h \omega_{f} .
$$

Let us compare obtained results with quantum electrodynamics formulas for the first radiation harmonic $(f=1)$ [7].

The results for energy of the first harmonic of scattered radiation depending on initial electron energy are coincided completely for both approaches. Fig. 2 shows energy of the scattered radiation depending on interaction angle for initial electron beam energy equal to $100 \mathrm{MeV}$. As one can see the coincidence of the results is practically ideal. But under small interaction angles (electron and wave move in the same direction) there is a difference in radiation energy value was caused by the fact that formulas [7] did not take into account relativistic factor $\beta$. Such consideration was carried out in [8]. The natural result is that under zero interaction angle the energy of scattered radiation is equal to initial wave energy. 


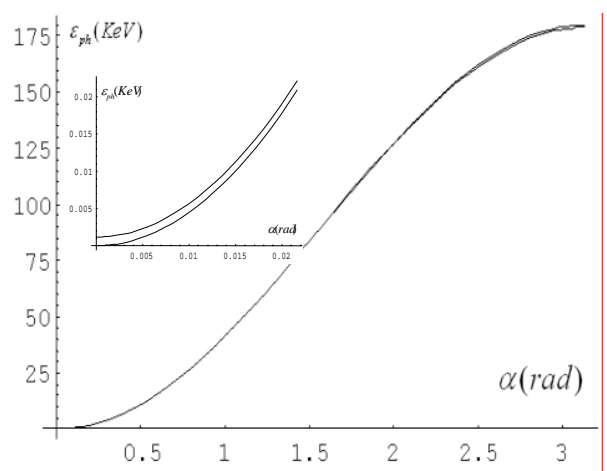

Figure 2. The energy of the first harmonic of scattered radiation depending on interaction angle $\left(\mathrm{E}_{0}=100 \mathrm{MeV}\right)$.

Fig. 3-4 show dependences of the energy of scattered radiation on initial energy of interacting electromagnetic wave and phase of interaction for the following parameters of interaction: photon beam radius $R=10^{-4} \mathrm{~m}$, laser flash length $L=10^{-2} \mathrm{~m}$, interaction angle equal to $\pi$, electron energy $E_{0}=100 \mathrm{MeV}$. With such parameters of laser flash the flash energy equal to $1 J$ will correspond to electric field strength $1.89 * 10^{10} \mathrm{~V} / \mathrm{m}$. As one can see these dependences become essential only for very intense initial waves.

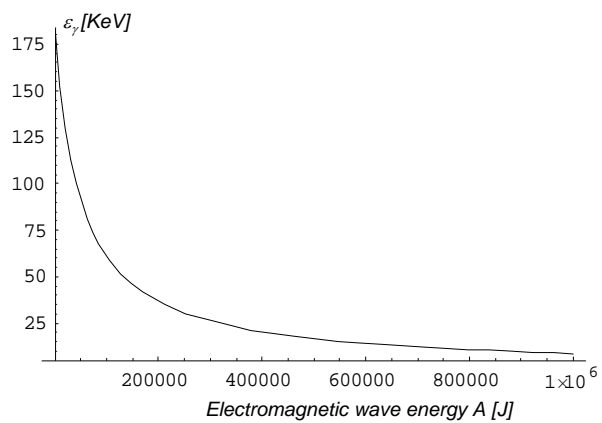

Figure 3. The energy of the first harmonic of scattered radiation depending on intensity of initial wave.

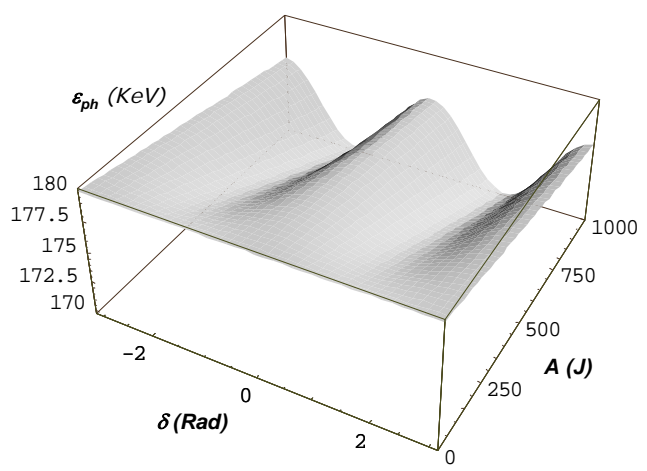

Figure 4. The energy of the first harmonic of scattered radiation depending on intensity of initial wave and phase of interaction (interaction angle equal to $\pi, \mathrm{E}_{0}=100 \mathrm{MeV}$ ).

To determine intensity of the radiation we will use the following well known expression [6]:

$$
\mathrm{d} \mathrm{I} / \mathrm{d} \Omega=\frac{c}{4 \pi}\left|H^{2}\right| R_{0}^{2} .
$$

It is needed to make integration in expression (14). And then, we change over direction to observer angles $\gamma_{1}, \gamma_{2}$ and $\gamma_{3}$ in the laboratory coordinate frame to angles in spherical coordinate frame $\theta$ and $\varphi$. Thus, substituting square of module from expression (15) and using expression (20), we obtain radiation intensity. Dependence of scattered radiation intensity on observer angles is shown on Fig. 5 for the interacting beam parameters mentioned above and laser beam intensity equal to $A=0.001 \mathrm{~J}$.

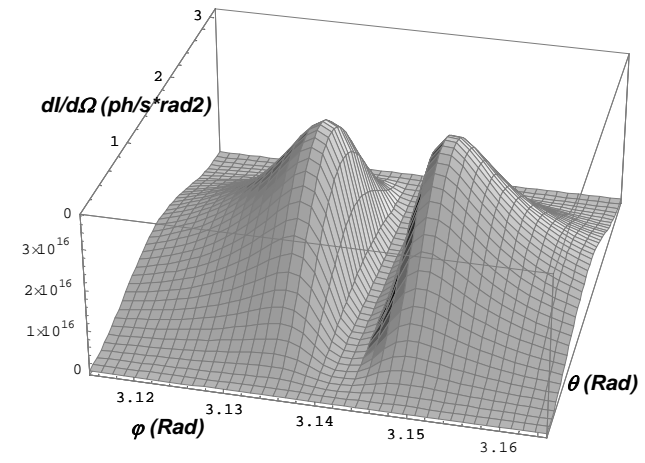

Figure 5. The scattered radiation intensity depending on observer angles (interaction angle equal to $\pi, \mathrm{E}_{0}=100$ $\mathrm{MeV}, \mathrm{A}=0.001 \mathrm{~J}, \mathrm{I}=0.01 \mathrm{~A}$ )

\section{CONCLUSION}

Thus, using classical electrodynamics approach we succeeded to obtain trajectories of an electron motion in the field of electromagnetic wave. Then, using a fact that obtained solutions are almost periodical functions we used generalized Fourier series and derived expressions for frequencies of the scattered radiation harmonics and spectrum of the radiation intensity. Obtained expressions allow investigating as well electron beam dynamics in laser electron X-ray generators as dependences of generated radiation parameters.

\section{REFERENCES}

[1] E. Bulyak, P. Gladkikh, A. Zelinsky et al. "Compact X-ray source based on Compton scattering", Nucl. Inst. \& Methods. 2002, A, № 487, p. 241-248.

[2] F.V. Hartemann, S. Anderson, C.P.J. Barty et al. "Ultrafast Compton Scattering X-Ray Source Development at LLNL", Proc. of EPAC 2004, 2004, p. 270-272.

[3] F.R. Arutyunian, V.A. Tumanian. "Compton effect on relativistic electrons and the possibility of obtaining beams of hard $\gamma$-quanta", JETP, 1963, v. 44, p. 2100-2103.

[4] V.I. Ritus. Quantum Electrodynamics of phenomena in intense field // Proceeding of Lebedev Physical Institute, 1979, v.3, 151 p.

[5] .Kh.Khokonov, M.Kh.Khokonov, R.M.Keshev. "High intensity limit in electron-laser beam interaction", Nucl. Inst. \& Methods. 2002, A, № 483, p. 998.

[6] L.D. Landau and E.M. Lifshits. "A field theory", 1967, Science, Moscow, 458 p.

[7] I. Ginzburg, G. Kotkin, V. Serbo, V. Telnov. "Colliding $\gamma e$ and $\gamma \gamma$ beams based on the single-pass $\mathrm{e}^{+} \mathrm{e}^{-}$colliders (VLEPP type)", Nucl. Inst. \& Methods. 1983, A, № 205, p. 47-69.

[8] E. Bulyak, V. Skomorokhov. "Parameters of Compton X-ray beam: Total yeld and pulse duration“, Phys. Rev. ST - AB 8, 030703, 2005. 